Objectives: To compare the ability of FRAX and BMD alone in discriminating patients with and without fracture, and their effectiveness in identifying antiosteoporotic treatment needs

Methods: 1300 rheumatic disease patients on long-term glucocorticoid were screened for vertebral fracture by radiograph from 7 rheumatology clinics. 220 ambulatory patients ( 110 with vertebral fracture and 110 without vertebral fracture) were recruited and assessed on CRF with FRAX questionnaire, BMD at the lumbar spine, total hip and femoral neck by dual-energy $x$-ray absorptiometry (DXA). The ten-year probability of MOF and hip fracture (FRAX score) with and without femoral neck BMD were calculated with the WHO FRAX tool.

Results: Both groups of patients were matched in gender, disease type and cumulative glucocorticoid dose $(p>0.1)$. Patients in the fracture group were older ( $63 \pm 14$ vs $59 \pm 12$ years, $p<0.01$ ), of lower body mass index $[22.7 \pm 3.5 \mathrm{vs}$ $\left.24 \pm 3.7 \mathrm{~kg} / \mathrm{m}^{2}, p<0.05\right]$, with a higher prevalence of previous fracture [38 $(34.5 \%)$ vs $5(4.5 \%), p<0.01]$, use of vitamin $D[77(70 \%)$ vs $54(49.1 \%), p<0.01]$ and bisphosphonates [23 (20.9\%) vs $7(6.4 \%), p<0.01]$. A receiver operating characteristic (ROC) curve analysis was performed to determine the ability of FRAX score with or without BMD and BMD alone to discriminate fracture status (Table 1). BMD at the femoral neck and FRAX score with BMD were able to provide adequate discrimination with their area under curve (AUC) $>0.70$. With regards to treatment indication, only $53 / 110(48.2 \%)$ of the patients with vertebral fracture were identified as having osteoporosis (T-score $\leq-2.5$ at hip/spine) by DXA. In contrast, FRAX with BMD were able to identify $71 / 110(64.5 \%)$ of the fracture patients whom treatment criteria were met, with a power of 0.97 and effect size of 0.38 (a medium effect) at the 0.05 level.

Table 1. Area under ROC curve on discriminating fracture

\begin{tabular}{|c|c|c|c|}
\hline & AUC & 95\% Confidence Interval \\
\hline \multicolumn{2}{|c|}{ BMD at Femoral Neck } & 0.709 & $0.640-0.778^{*}$ \\
\hline \multicolumn{2}{|c|}{ BMD of Total Hip } & 0.693 & $0.623-0.763^{*}$ \\
\hline \multicolumn{2}{|c|}{ BMD of Lumbar Spine } & 0.654 & $0.581-0.727^{*}$ \\
\hline \multirow[t]{2}{*}{ FRAX with BMD } & MOF & 0.705 & $0.635-0.775^{*}$ \\
\hline & Hip fracture & 0.720 & $0.651-0.789^{*}$ \\
\hline FRAX without & MOF & 0.681 & $0.610-0.752 *$ \\
\hline BMD & Hip fracture & 0.687 & $0.616-0.758^{*}$ \\
\hline
\end{tabular}

Conclusions: In rheumatic patients on long-term glucocorticoid, FRAX score was more effective in identifying patients with intervention needs than BMD alone. It is suggested that FRAX should be used as a routine assessment to identify patients, even asymptomatic, indicated for anti-osteoporotic treatment.

References:

[1] Aspray, T. J. (2015). Fragility fracture: recent developments in risk assessment. Therapeutic Advances in Musculoskeletal Disease, 7(1), 17-25. http://doi.org/10.1177/1759720X14564562.

[2] Rentero, M. L., Amigo, E., Chozas, N., Fernández Prada, M., Silva-Fernández, L., Abad Hernandez, M. A., ... del Pino-Montes, J. (2015). Prevalence of fractures in women with rheumatoid arthritis and/or systemic lupus erythematosus on chronic glucocorticoid therapy. BMC Musculoskeletal Disorders, 16(1), 300. article. https://doi.org/10.1186/s12891-015-0733-9.

Acknowledgements: We would like to acknowledge the Research Grant Council of Hong Kong for funding support (RGC Ref No.14113714).

Disclosure of Interest: None declared

DOI: 10.1136/annrheumdis-2017-eular.3591

\section{FRI0577 CLINICAL CHARACTERISTICS OF SPONTANEUS FRACTURES IN THE BEDRIDDEN PATIENTS UNINTENTIONALLY CAUSED BY CAREGIVERS}

T. Kashiwagura ${ }^{1}$, Y. Kimura ${ }^{1}$, I. Wakabayashi ${ }^{2}$, M. Fujii ${ }^{1}$, K. Nozaka ${ }^{3}$, N. Miyakoshi ${ }^{3}$, Y. Shimada ${ }^{3} .{ }^{1}$ Department of Orthopedic Surgery; ${ }^{2}$ Department of Rehabilitation Medicine, Akita City Hospital; ${ }^{3}$ Department of Orthopedic Surgery, Akita University Graduate School of Medicine, Akita, Japan

Background: The number of bedridden patients in our super-aging society is increasing. We previously studied fractures occurring in bedridden patients during daily activities with caregiver assistance (dry baths, range of motion exercises, transfer to wheelchair, changing diapers, and so forth) and non-traumatic fractures detected by pain, swelling, subcutaneous bleeding, deformity, and so on. These minimal trauma or spontaneous fractures were defined as "spontaneus fractures in the bedridden patients unintentionally caused by caregivers". Despite efforts to draw attention to preventing these injuries, the incidence of such fractures has been increasing. Spontaneus fractures caused by caregivers are characterized by the presence of untreated osteoporosis, contracture or spasticity near the lesion, institutionalization or hospitalization, repeated fractures, femur fracture, and other unknown causes.

Objectives: Spontaneus fractures caused by caregivers frequently occur without any noticeable trauma and at present, predicting the risk of occurrence is difficult. This study aimed to explore the usefulness of bone metabolism markers in predicting the risk for spontaneus fractures caused by caregivers.

Methods: Study subjects were selected from a pool of 28 patients ( 3 men; 25 women) with 33 spontaneus fractures caused by caregivers who were treated in our hospital between April 2006 and July 2016. Patients were selected based on the following inclusion criteria: no renal dysfunction $($ GFR $\geq 60$ ) and those who had undergone measurement of bone metabolism markers. Finally, 12 women with a mean age at onset of $90.6(76-100)$ years were enrolled in the study. The following markers were evaluated: the bone formation markers bone type alkaline phosphatase and intact procollagen type 1 amino-terminal propeptide; the bone resorption markers tartrate-resistant acid phosphatase (TRACP)-5b, urinary deoxypyridinoline (DPD), and serum $\mathrm{N}$-telopeptide; and the bone quality markers undercarboxylated osteocalcin (uCOC), urinary pentosidine (Pen), and homocysteine (Hcy).

Results: Low levels of bone metabolism markers were not observed in these subjects. However, TRACP-5b levels were high in six subjects while urinary DPD levels were abnormally high in all subjects with a mean value of 23.3 (9.2-41.4) $\mathrm{nmol} / \mathrm{mmol}$ creatinine $(\mathrm{Cr})$. With regards to bone quality markers, levels of Hcy and $\mathrm{uCOC}$ were high in two and three subjects, respectively; while Pen levels were abnormally high in ten subjects with a mean value of $0.183(0.0774-0.3115)$ $\mu \mathrm{g} / \mathrm{mg} \mathrm{Cr}$.

Conclusions: The majority of subjects with spontaneus fractures caused by caregivers had untreated osteoporosis and some had repeated fractures, indicating that early treatment of osteoporosis is important. Assessing osteoporosis in bedridden patients is challenging as measurement of bone density is often difficult due to spinal deformity and contracture. Our results suggest that among the bone metabolism markers, DPD and Pen may be useful predictors for the risk of spontaneus fractures caused by caregivers.

\section{References:}

[1] T Kashiwagura et al. Clinical results of spontaneous fractures in the bedridden patients unintentionally caused by caregivers. Orthop Surg Traumatol 56:189193, 2013.

Acknowledgements: The authors would like to thank Y. Sasaki for technical assistance.

Disclosure of Interest: None declared

DOI: 10.1136/annrheumdis-2017-eular.4531

\section{FRI0578 ODONTOID FRACTURES IN THE ELDERLY: AN UNKNOWN OSTEOPOROTIC FRACTURE?}

L.L. Natella ${ }^{1}$, N. Bronsard ${ }^{2}$, J. Allia ${ }^{2}$, L. Hekayem ${ }^{3}$, L. Euller-Ziegler ${ }^{1}$, F. De Peretti $^{2}, \underline{V}$. Breuil ${ }^{1}$. ${ }^{1}$ Rheumatology/University of Nice, Rheumatology/University of Nice; ${ }^{2}$ Department of Orthopedic Surgery; ${ }^{3}$ Emergency Room Department, University of Nice, nice, France

Background: Current WHO definition of osteoporosis excludes cervical fractures. However, in atraumatic odontoid fractures, mainly reported by orthopedic surgeons, bone status has not been described yet [1].

Objectives: To investigate bone status in elderly patients sustaining a low energy odontoid fracture.

Methods: We conducted a prospective study from January 2016 to January 2017 in patients $>65$ years old, hospitalized in Nice University hospital for low energy odontoid fracture. An evaluation of bone status was proposed within 3 months after fracture event. Evaluation included demographic data, clinical risk factors of osteoporosis, bone mineral density (BMD) at spine and hip and vertebral fracture assessment (VFA) by dual X-ray absorptiometry and serum analysis to detect secondary osteoporosis.

Results: 38 patients were hospitalized for odontoid fracture: 8 patients $<65$ years always after a major trauma (mean age $37.1 \pm 14.5 \mathrm{y}$ ) and 30 patients $\geq 65$ years including 3 after a high energy impact. 27 odontoid fractures followed a low energy impact: 18 women and 9 men, mean age 83.8 y. $( \pm 10.7) .8$ patients died before bone status assessment ( 5 men and 3 women), 6 died during hospitalization with a mean delay of 3.5 days $( \pm 1.87)$ and 2 after discharge ( 1 month and 5 month). 3 patients refused bone status evaluation, 5 were lost to follow-up and 1 is awaiting evaluation. Finally 10 patients had bone status evaluation, all women, mean age 84.2 y. $( \pm 8.9)$. None had parental history of hip fracture, 1 had an early menopause, 1 received aromatase inhibitors for breast cancer and 2 had a history of steroid therapy ( $>3$ months). 3 patients had previously received hormone replacement therapy, 1 received bisphosphonate for 5 years and 4 had calcium + vitamin D supplements. Lumbar spine mean T-score was $-1.45( \pm 1.08)$, femoral neck: $-2.37( \pm 0.040)$ and total hip: $-1.99( \pm 0.6)$. VFA analysis revealed 4 unknown vertebral fractures. The table summarizes population bone status: 8 patients out of 10 fulfilled diagnostic criteria of osteoporosis, including 6 with previous fractures. 2 patients with T-score > -1.DS didn't have hip BMD assessment because of bilateral hip replacement but had previous major osteoporotic fractures. No

\begin{tabular}{|l|l|l|l|}
\hline \multicolumn{1}{|c|}{ Lowest T-score } & No fracture (n) & $\begin{array}{c}\text { Previous minor } \\
\text { osteoporotic fracture }(\mathrm{n})\end{array}$ & Previous major osteoporotic fracture $(\mathrm{n})$ \\
\hline T-score $>-1$ DS & 0 & 0 & $\begin{array}{l}2 \\
\text { (1 patient. } 2 \text { femoral neck fracture, } \\
1 \text { patient: pelvic and humeral fractures. } \\
\text { Both patients had vertebral fracture on VFA })\end{array}$ \\
\hline-1 DS $>$ T-score $2-2.5$ & 1 & $1(1$ wrist) & $\begin{array}{l}2 \\
(1 \text { patient humeral and vertebral fracture on } \\
\text { VFA, } \\
1 \text { patient. } 5 \text { ribs fractures })\end{array}$ \\
\hline T-score $<-2.5$ & 2 & $1(2$ wrist fractures) & 1 (vertebral fracture on VFA) \\
\hline
\end{tabular}

